\title{
A Rare Case of Embolization of a PFO Occluder Device to the Abdominal Aorta in a Patient with Bicuspid Aortic Valve and Previous Aortic Coarctation: A Case Report
}

\author{
Luca Allievi $^{1}$, Amedeo Bongarzoni ${ }^{2}$, Guido Tassinario ${ }^{2}$, and Stefano Carugo ${ }^{1}$ \\ ${ }^{1}$ Fondazione IRCCS Ca' Granda Ospedale Maggiore Policlinico \\ ${ }^{2}$ ASST Santi Paolo e Carlo
}

February 7, 2021

\begin{abstract}
Patent foramen ovale (PFO) is a common congenital defect in adults; its closure with an occluder device is usually a safe procedure. However, a rare and potentially fatal complication is the device embolization. We report a case of a 52-year-old man with bicuspid aortic valve and surgically corrected aortic coarctation in which a PFO occluder device migrated to the abdominal aorta. We recommend paying attention to the risk factors before the intervention (PFO characteristics and multiple congenital defects) and to the clinical-echocardiographic follow-up, to prevent, recognize and treat this severe complication as soon as possible.
\end{abstract}

\section{A Rare Case of Embolization of a PFO Occluder Device to the Abdominal Aorta in a Patient with Bicuspid Aortic Valve and Previous Aortic Coarctation: A Case Report}

Running Head: Rare Embolization of a PFO Occluder Device

Authors: Luca Allievi ${ }^{1, *}, \mathrm{MD}$; Amedeo Bongarzoni ${ }^{2}, \mathrm{MD}$; Guido Tassinario ${ }^{2}, \mathrm{MD}$; Stefano Carugo ${ }^{1}$, MD.

${ }^{1}$ Department of Cardiology, Fondazione IRCCS Ca' Granda Ospedale Maggiore Policlinico, University of Milan, 20122 Milan, Italy.

${ }^{2}$ Department of Cardiology, ASST Santi Paolo e Carlo, University of Milan, 20142 Milan, Italy.

*Corresponding author: Luca Allievi, MD, Department of Cardiology, Fondazione IRCCS Ca' Granda Ospedale Maggiore Policlinico, Via Sforza 35, University of Milan, 20122 Milan, Italy, Phone: 0039-0255033517, E-mail:luca.allievi@unimi.it, Fax: none.

Abstract: Patent foramen ovale (PFO) is a common congenital defect in adults; its closure with an occluder device is usually a safe procedure. However, a rare and potentially fatal complication is the device embolization. We report a case of a 52-year-old man with bicuspid aortic valve and surgically corrected aortic coarctation in which a PFO occluder device migrated to the abdominal aorta. We recommend paying attention to the risk factors before the intervention (PFO characteristics and multiple congenital defects) and to the clinical-echocardiographic follow-up, to prevent, recognize and treat this severe complication as soon as possible.

Keywords : aorta coarctation; patent foramen ovale; atrial septal aneurysm (ASA); transcatheter closure device; bicuspid aortic valve; echocardiography.

\section{Introduction}


Patent Foramen Ovale (PFO) is a congenital intracardiac defect present in about $25 \%$ of the general population, due to incomplete fusion of septum primum and secundum after birth. It predisposes to paradoxical embolism, in which emboli from the peripheral venous system provoke systemic strokes or infarcts. Given the low incidence of paradoxical embolism $(1 \% \text { per year })^{1}$, percutaneous PFO closure is performed only in presence of a thromboembolic event (e.g. stroke) caused with high probability by the presence of a PFO associated with a high risk of recurrence ${ }^{2}$. After the procedure, several complications can occur: haemorrhage, pulmonary embolism, infective endocarditis, cardiac perforation and device erosion, fracture, thrombosis or embolization $^{3}$. Device embolization is very rare (0.1-0.4\%) but potentially life-threatening, and requires immediate removal of the embolized device ${ }^{3}$. Possible migration sites are: pulmonary artery, heart chambers, aortic arch, descending thoracic and abdominal aorta ${ }^{4}$. We report below a case of embolization of a PFO occluder device into the abdominal aorta.

\section{Case Presentation}

A 52-year-old male patient came to our Echocardiography Laboratory at the San Carlo Hospital in Milan on $31^{\text {st }}$ December 2020, following the onset of dyspnoea with light exertion and chest pain radiated to both upper limbs. He denied having cardiovascular risk factors, but reported being a carrier of bicuspid aortic valve (BAV) and having been operated for aortic coarctation (AC) at the age of 14 . In June 2020, he had been admitted to another hospital Neurology ward for an ischemic stroke of the vertebrobasilar circulation; the transthoracic echocardiogram (TTE) had shown a slight spontaneous left-right shunt and positive bubblestudy, with final diagnosis of PFO. In October 2020, percutaneous PFO closure by endovascular positioning of an Occlutech device $(23 / 25 \mathrm{~mm}$ ) had been performed; then, he had been discharged on dual antiplatelet and statin therapy without performing a TTE before discharge, only suggesting to perform a TTE with bubble study 4-6 months after the intervention. About 7 days after discharge, upon resuming work (employee in septic service), he started feeling shortness of breath with light efforts and chest pain radiated to the upper limbs (described as myalgia) everyday but only upon awakening. For this reason, he went to our Laboratory for a physical examination with TTE. The physical examination was normal, while the only ECG finding was a right bundle branch block. The TTE showed the absence of the occluder device in place, with spontaneous left-right shunt (Fig. 1); in addition, the transoesophageal echocardiogram (TEE) detected the absence of the device, an ASA and a long tunnel (Fig. 2). A chest/abdomen CT scan with contrast showed the device into the abdominal aorta, at the first lumbar vertebra (L1), near the origin of the coeliac trunk (from which the superior mesenteric artery also arose) and just proximal to the origin of the renal arteries (Fig. 3). An abdominal aorta ultrasound showed the device completely intact (two hemidiscs), blocked into the aortic lumen at the origin of the coeliac trunk (Fig. 4): the device partially occluded both vessels without causing gastrointestinal or lower limb symptoms. The patient was then admitted to our Cardiology ward, still presenting myalgias during the night and in the morning (no fever, negative Sars-Cov-2 RT-PCR swab test, normal creatine kinase). Finally, the device retrieval was performed through an hybrid percutaneous and surgical procedure at the referral centre on $5^{\text {th }}$ January 2021. Through right femoral access, the device was captured and pulled with a $35 \mathrm{~mm}$ Goose Neck snare (Fig. 5) and then with a $50 \mathrm{~cm}$ bioptome, however losing the device twice. Therefore, the device was finally surgically removed from the right femoral artery. After the device removal, the patient felt no more myalgias. A new PFO closure with Noblestitch was proposed to the patient but he decided not to underwent another procedure for at least 1-2 months.

\section{Discussion}

\section{Risk Factors}

Predisposing factors for device embolization may be: device too small, atrial septal aneurysm (ASA), thick septum secundum $(>10 \mathrm{~mm})$, long tunnel ${ }^{5}([?] 8 \mathrm{~mm})^{6}$, deficient rims of surrounding tissue and device malpositioning ${ }^{3}$. In this case, we believe that the choice of a device too small, the presence of an ASA and a long tunnel could have favoured the device leaving from its site. The PFO tunnel was longer than measured at the TEE during the intervention (24 mm vs. $13-14 \mathrm{~mm}$, respectively): the possible tunnel length underestimation could have led to the choice of an unsuitable device for the PFO characteristics and, therefore, to its displacement. The patient denied having performed manoeuvres potentially able to facilitate 
the device displacement, such as having coughed heavily or repeatedly ${ }^{7,8}$. A peculiarity of this case is the finding of this complication in presence of other congenital defects (BAV and previous AC). The presence of other congenital defects may have predisposed the device displacement (e.g. precluding an adequate device fixation). Furthermore, the surgical repair of the aortic coarctation may have favoured a more distal drop of the device. The device embolization in presence of multiple congenital defects represents a unique event in the literature, which gives thought to other possible mechanisms of the PFO occluder device displacement in patients with multiple congenital defects.

\section{Symptoms}

The mechanisms underlying the symptoms remain unclear. We assume that the onset of dyspnoea after the intervention was caused by the worsening of the right-left shunt (provoked by exercise) due to the PFO enlargement following the device displacement; this may be supported by the detection of a significant oxygen desaturation on the 6-Minutes Walking Test (98\% to $92 \%$ after 2 minutes), an event already observed in other patients with PFO, defined as Provoked Exercise Desaturation (PED) ${ }^{9}$. We excluded Platypnea Orthodeoxia Syndrome (POS), as the patient had a normal arterial oxygen saturation in standing position. We also presume that the chest pain radiated to the limbs, after having excluded statin-induced myopathy (normal creatine kinase), was the expression of an aortic syndrome with atypical features, due to the presence of the foreign body into the abdominal aorta. This may be confirmed by its disappearance after the device retrieval.

\section{Follow-up}

Lastly, we believe that if a TTE before discharge or a TTE with bubble study within 1 month from discharge had been performed, the problem would certainly have been identified earlier and with lower risk for the patient. Indeed, the current guidelines recommend TTE before discharge, TTE with bubble study after $1,6,12$ months and, then, every 1-2 years ${ }^{3}$. However, a correct follow-up probably was hindered by the COVID-19 pandemic.

\section{Conclusion}

As shown, the embolization of a PFO occluder device is a very rare but possible complication (in this case reported for the first time in a patient with multiple congenital defects). For this reason, we strongly recommend paying attention to 1) risk factors before the intervention (PFO characteristics and multiple congenital defects) to correctly choose the device to be placed, and to 2) the clinical-echocardiographic follow-up to recognize and treat this severe complication as soon as possible.

\section{Statement of Concent}

Not necessary

\section{Competing Interests Statement}

None declared.

\section{Funding}

This research received no specific grant from any funding agency.

\section{Authors' contribution}

L.A.: Data collection, Concept/design, Literature review, Drafting article, Approval of article

A.B.: Data collection, Concept/design, Literature review, Drafting article, Approval of article

G.T.: Data collection, Concept/design, Literature review, Drafting article, Approval of article

S.C.: Concept/design, Literature review, Revising article, Approval of article

\section{References}


1. Messé, S. R. et al. Practice advisory update summary: Patent foramen ovale and secondary stroke prevention. Neurology (2020) doi:10.1212/wnl.0000000000009443.

2. Pristipino, C. et al. European position paper on the management of patients with patent foramen ovale. General approach and left circulation thromboembolism. Eur. Heart J. (2019) doi:10.1093/eurheartj/ehy649.

3. Silvestry, F. E. et al. Guidelines for the Echocardiographic Assessment of Atrial Septal Defect and Patent Foramen Ovale: From the American Society of Echocardiography and Society for Cardiac Angiography and Interventions. J. Am. Soc. Echocardiogr. (2015) doi:10.1016/j.echo.2015.05.015.

4. Mancini, M. E., Muratori, M., Agostoni, P. \& Trabattoni, D. When a patent foramen ovale device is no more in place: silent patent foramen ovale occluder device migration to the aortic arch. Eur. Hear. J. - Case Reports 3, (2019).

5. Goel, S. S., Aksoy, O., Tuzcu, E. M., Krasuski, R. A. \& Kapadia, S. R. Embolization of patent foramen ovale closure devices: Incidence, role of imaging in identification, potential causes, and management.Texas Hear. Inst. J. (2013).

6. Vitarelli, A. et al. Echocardiographic findings in simple and complex patent foramen ovale before and after transcatheter closure.Eur. Heart J. Cardiovasc. Imaging (2014) doi:10.1093/ehjci/jeu143.

7. Bartel, T., Bonatti, J. O. \& Müller, S. Device Dislocation, Probably Due to Paroxysmal Coughing Early After Percutaneous Closure of Secundum Type Atrial Septal Defect. Am. J. Cardiol. (2008) doi:10.1016/j.amjcard.2007.08.059.

8. Schoof, S., Norozi, K., Breymann, T., Wessel, A. \& Bertram, H. Cough a potentially lifethreatening condition after interventional closure of atrial septal defect. Circ. Cardiovasc. Imaging (2009) doi:10.1161/CIRCIMAGING.108.808873.

9. Devendra, G. P., Rane, A. A. \& Krasuski, R. A. Provoked exercise desaturation in patent foramen ovale and impact of percutaneous closure.JACC Cardiovasc. Interv. (2012) doi:10.1016/j.jcin.2012.01.011.

\section{Figure legend}

Figure 1. TTE performed from a 2D subcostal view demonstrating the absence of the PFO occluder device in the interatrial septum (Panel A) and the presence of a spontaneous left-to-right shunt due to PFO in CFM (Panel B). 2D, two dimensional; CFM, colour-flow mode; LA, left atrium; LV, left ventricle; PFO, patent foramen ovale; RA, right atrium; RV, right ventricle; TTE, transthoracic echocardiogram.

Figure 2. TEE subsequently performed confirming the absence of the occluder device in the interatrial septum and showing a very long PFO tunnel (at least $24 \mathrm{~mm}$ ). Ao, aorta; LA, left atrium; PFO, patent foramen ovale; RA, right atrium; PFO, patent foramen ovale; TEE, transoesophageal echocardiogram.

Figure 3. Thoracoabdominal CT scan from an axial (Panel A), sagittal (Panel B) and coronal plane (Panel C) showing the occluder device into the abdominal aorta at L1 vertebral level near the origin of the coeliac trunk. Ao, aorta; CT, computed tomography; L1, first lumbar vertebra.

Figure 4. Abdominal ultrasound demonstrating the device with its two hemidiscs into the aortic lumen (Panel A), blocked at the origin of the coeliac trunk as seen in CFM (Panel B). Ao, aorta; CFM, colour-flow mode.

Figure 5. Fluoroscopic image showing the percutaneous retrieval of the device with a $35 \mathrm{~mm}$ Goose Neck snare. The procedure was partially successful and a resolutive surgical procedure was needed. 


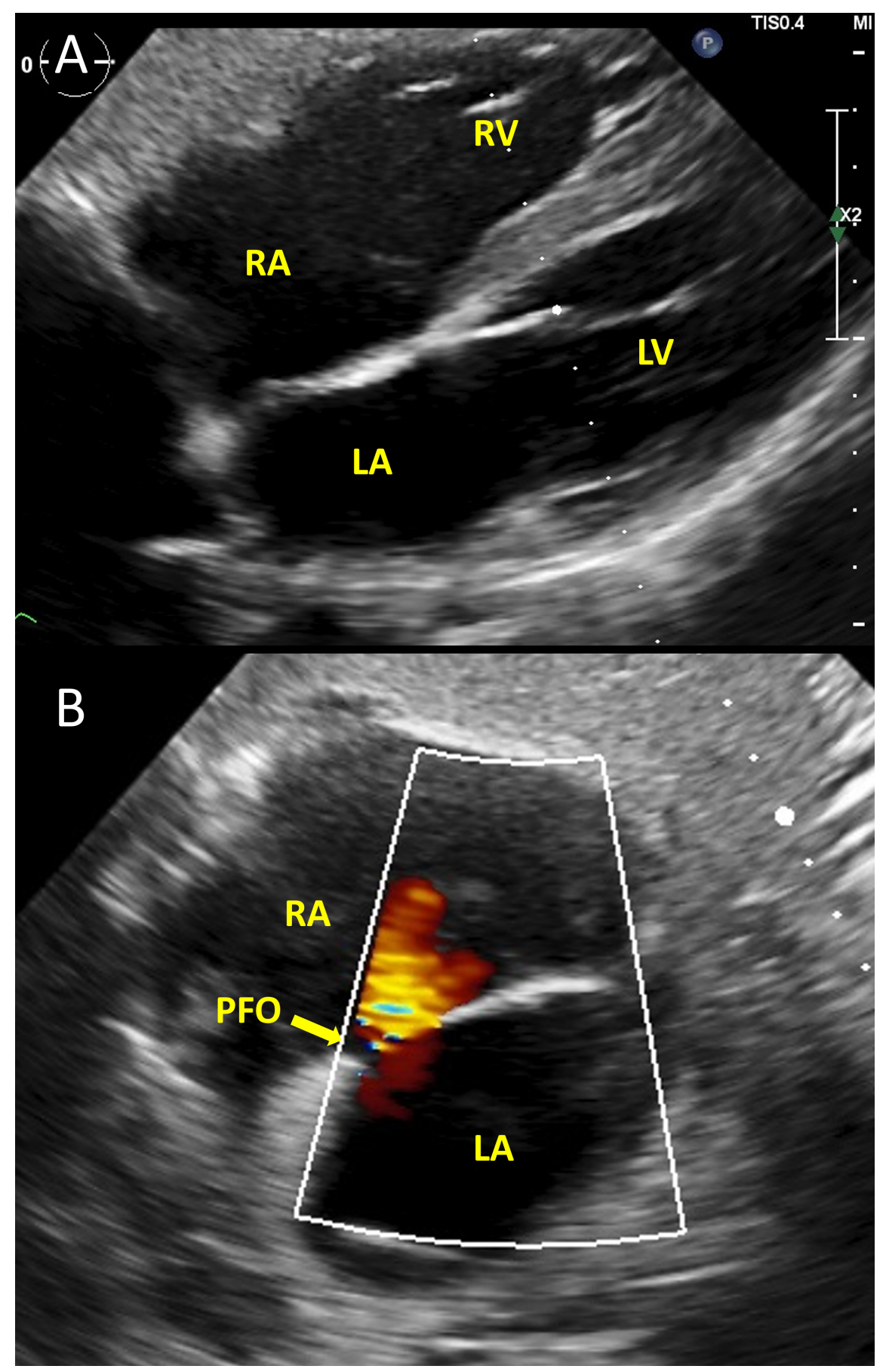




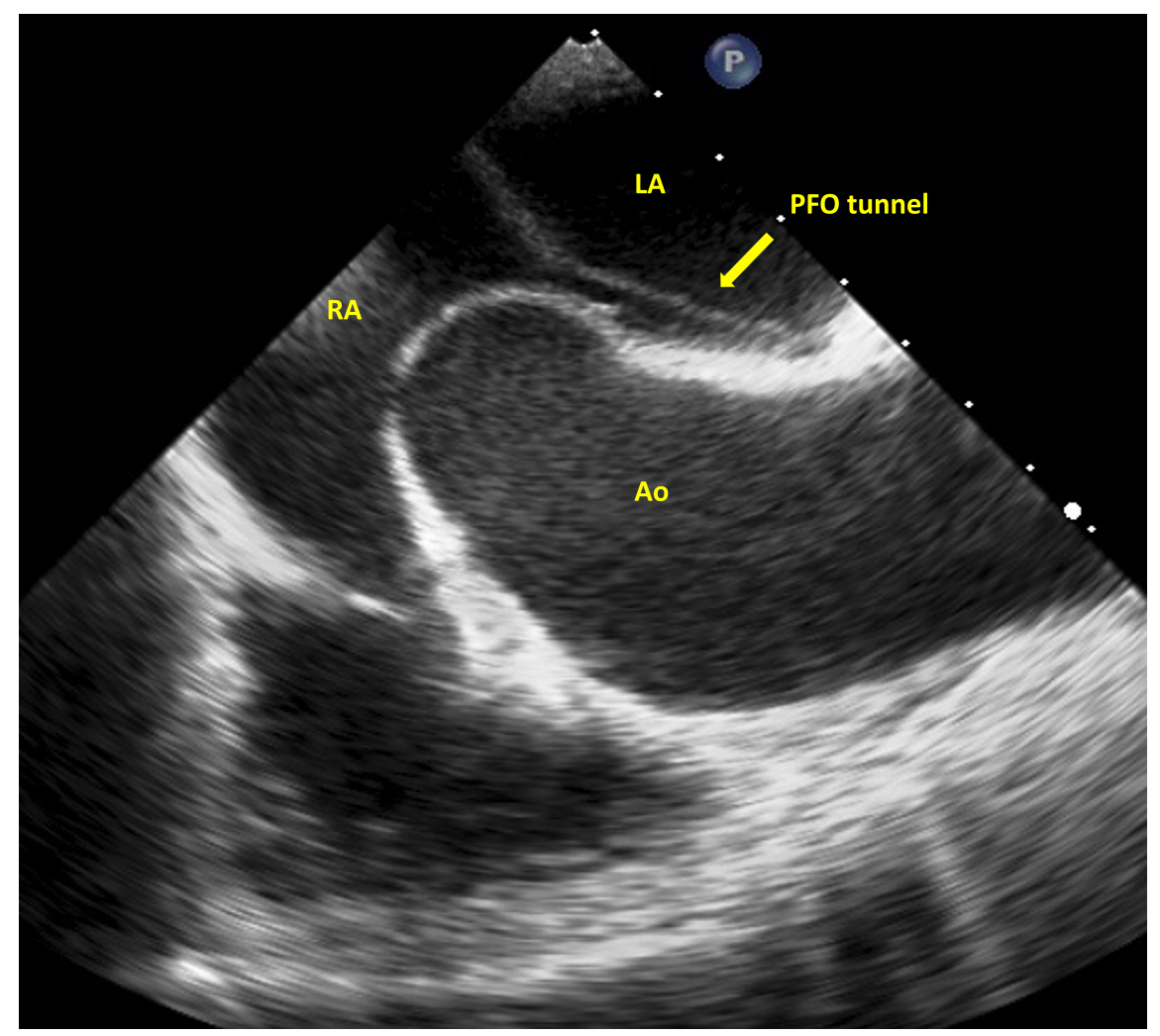




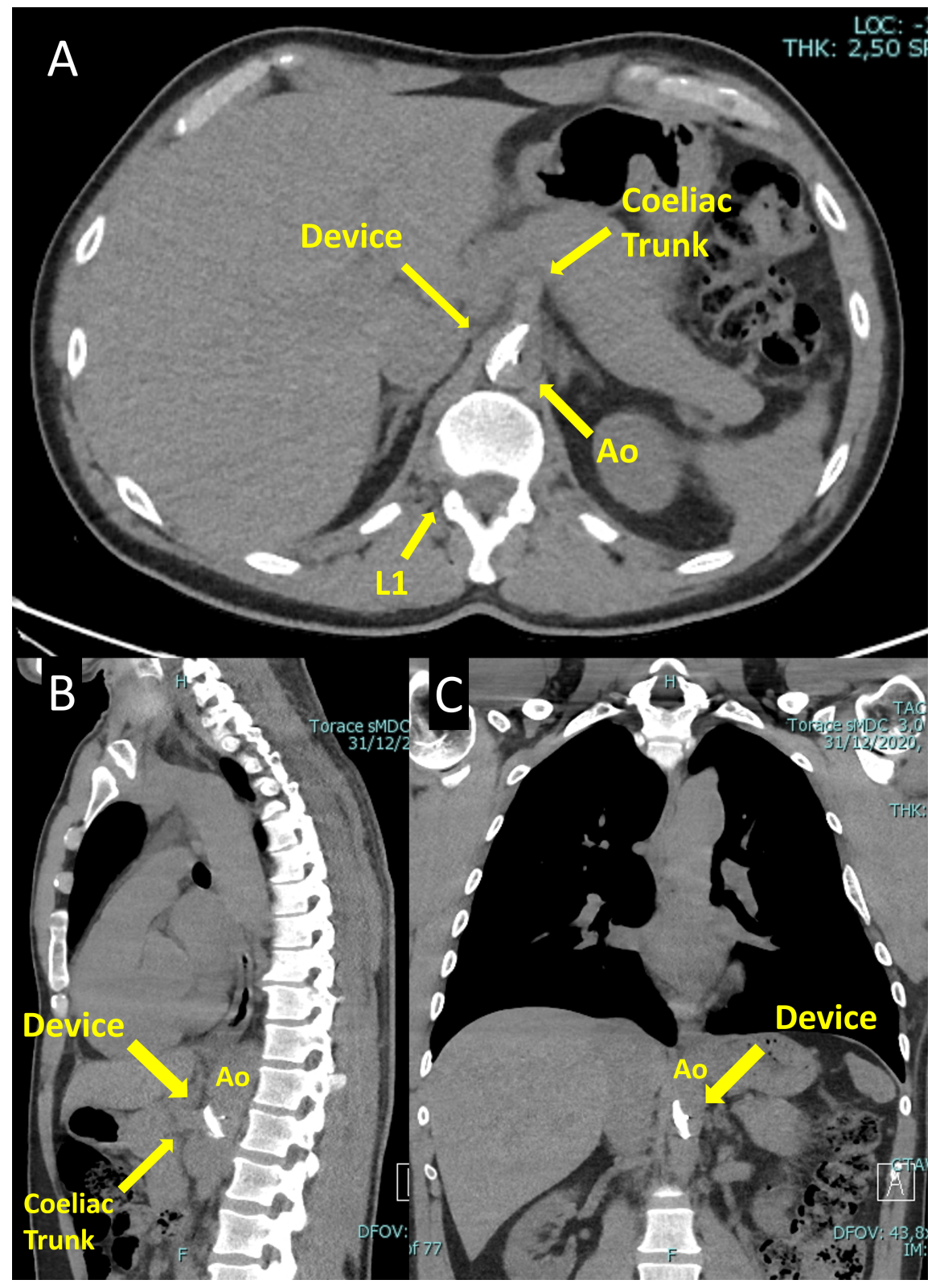




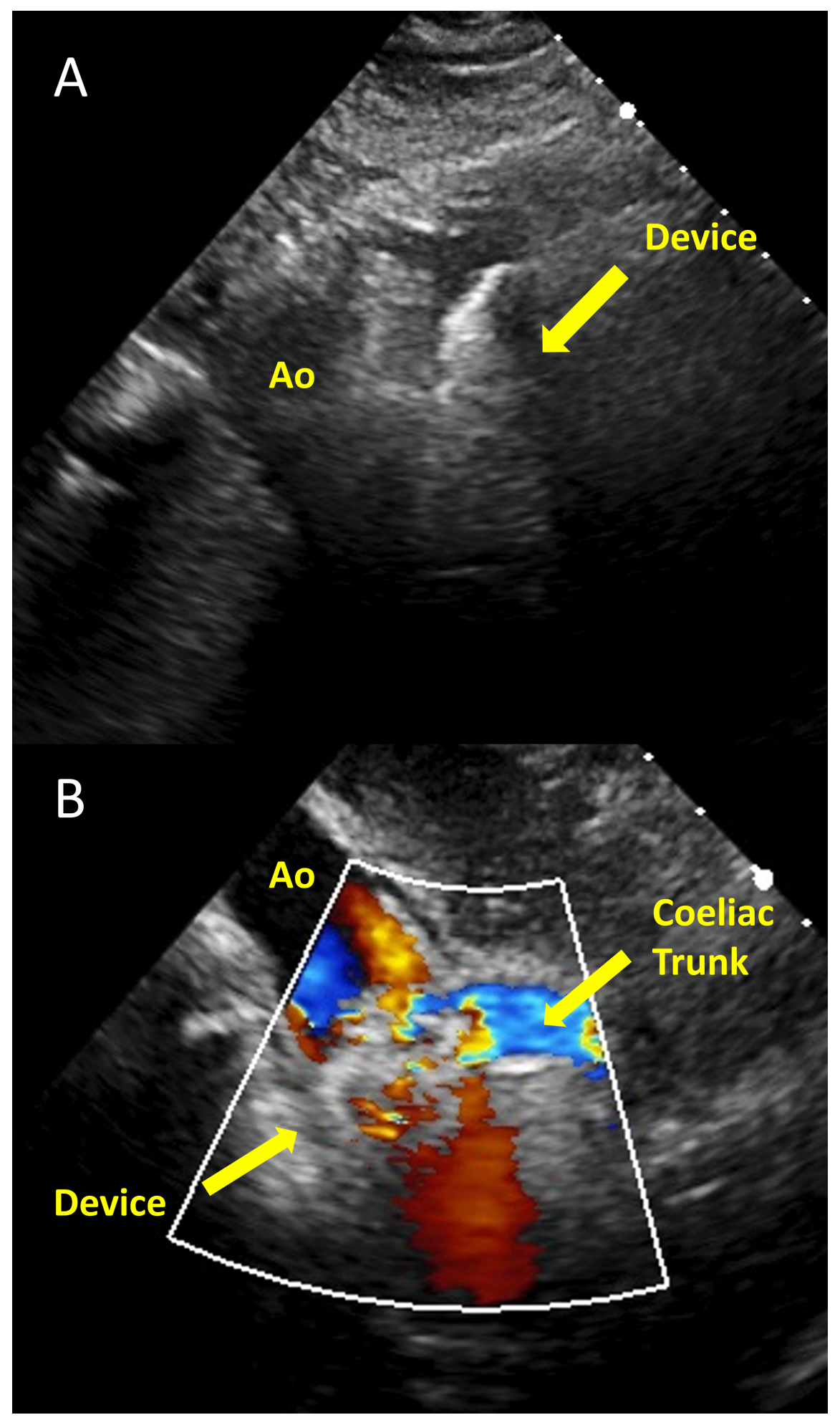




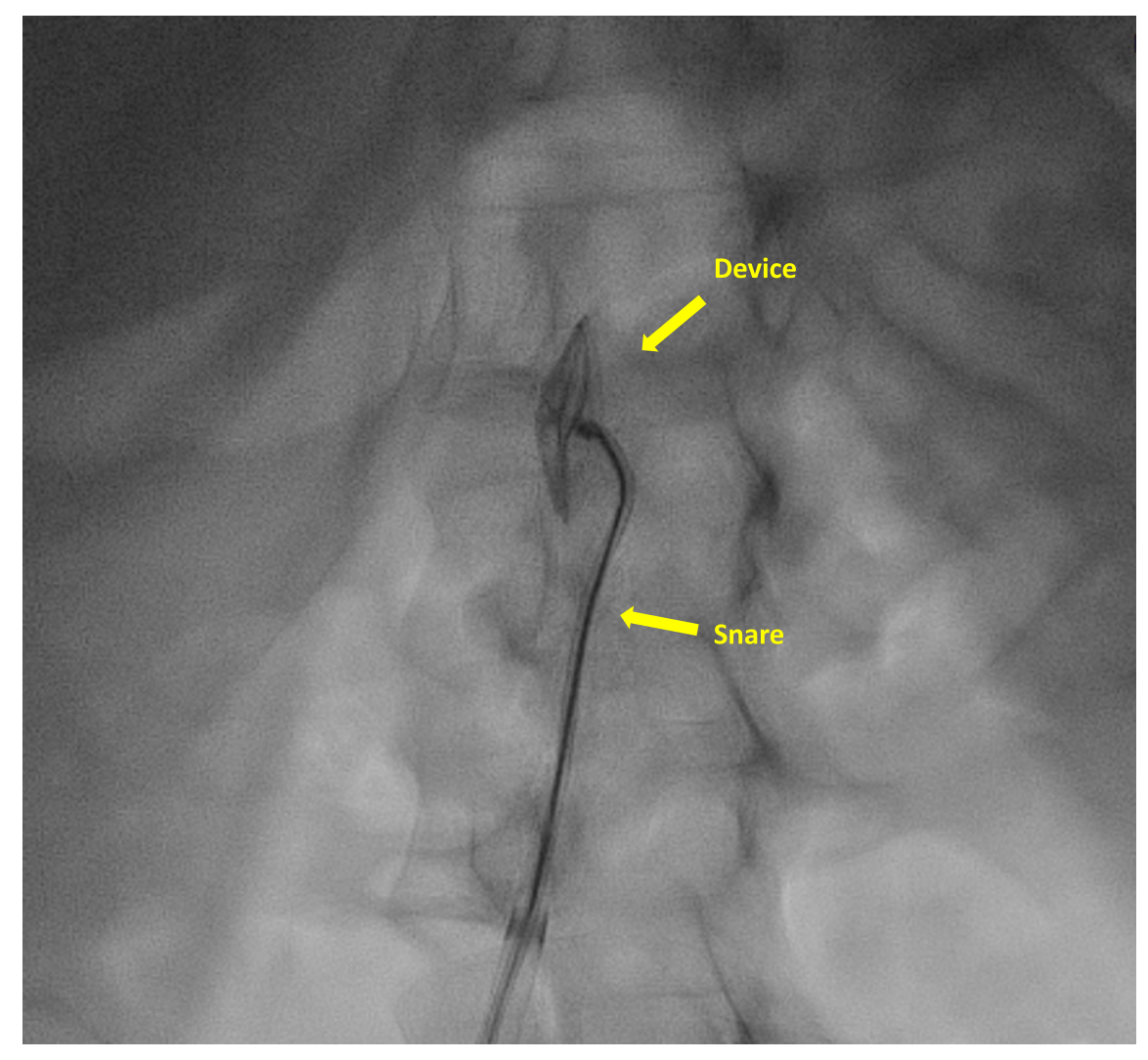

